\title{
The joint distribution of $q$-additive functions
}

\author{
by \\ Michael Drmota (Wien)
}

1. Introduction. Let $q>1$ be a given integer. A real-valued function $f$, defined on the non-negative integers, is said to be $q$-additive if $f(0)=0$ and

$$
f(n)=\sum_{j \geq 0} f\left(a_{q, j}(n) q^{j}\right) \quad \text { for } \quad n=\sum_{j \geq 0} a_{q, j}(n) q^{j},
$$

where $a_{q, j}(n) \in E_{q}:=\{0,1, \ldots, q-1\}$. A special $q$-additive function is the sum-of-digits function

$$
s_{q}(n)=\sum_{j \geq 0} a_{q, j}(n) .
$$

The statistical behaviour of the sum-of-digits function and, more generally, of $q$-additive functions has been very well studied by several authors.

The most general result concerning the mean value of $q$-additive functions is due to Manstavičius [20] (extending earlier work of Coquet [3]). Let

$$
m_{k, q}:=\frac{1}{q} \sum_{c \in E_{q}} f\left(c q^{k}\right), \quad m_{2 ; k, q}^{2}:=\frac{1}{q} \sum_{c \in E_{q}} f^{2}\left(c q^{k}\right)
$$

and

$$
M_{q}(x):=\sum_{k=0}^{\left[\log _{q} x\right]} m_{k, q}, \quad B_{q}^{2}(x)=\sum_{k=0}^{\left[\log _{q} x\right]} m_{2 ; k, q}^{2} .
$$

Then

$$
\frac{1}{x} \sum_{n<x}\left(f(n)-M_{q}(x)\right)^{2} \leq c B_{q}^{2}(x),
$$

2000 Mathematics Subject Classification: Primary 11A63; Secondary 11N60.

This research was supported by the Austrian Science Foundation FWF, grant S8302MAT. 
which implies

$$
\frac{1}{x} \sum_{n<x} f(n)=M_{q}(x)+O\left(B_{q}(x)\right) .
$$

For the sum-of-digits function $s_{q}(n)$ much more precise results are known, e.g. Delange [5] proved (for integral $x$ ) that

$$
\frac{1}{x} \sum_{n<x} s_{q}(n)=\frac{q-1}{2} \log _{q} x+\gamma\left(\log _{q} x\right),
$$

where $\gamma$ is a continuous, nowhere differentiable and periodic function with period 1. (Higher moments of $a_{q}(n)$ were considered by Kirschenhofer [19] and by Kennedy and Cooper [17] (for the variance) and by Grabner, Kirschenhofer, Prodinger and Tichy [12].)

There also exist distributional results for $q$-additive functions. In 1972 Delange [4] proved an analogue to the Erdős-Wintner theorem. There exists a distribution function $F(y)$ such that, as $x \rightarrow \infty$,

$$
\frac{1}{x} \#\{n<x \mid f(n)<y\} \rightarrow F(y)
$$

if and only if the two series $\sum_{k>0} m_{k, q}, \sum_{k>0} m_{2 ; k, q}^{2}$ converge. This theorem was generalized by Kátai [16] who proved that there exists a distribution function $F(y)$ such that, as $x \rightarrow \infty$,

$$
\frac{1}{x} \#\left\{n<x \mid f(n)-M_{q}(x)<y\right\} \rightarrow F(y)
$$

if and only if the series $\sum_{k \geq 0} m_{2 ; k, q}^{2}$ converges.

The most general theorem known concerning a central limit theorem is again due to Manstavičius [20]. Suppose that, as $x \rightarrow \infty$,

$$
\max _{c q^{j}<x}\left|f\left(c q^{j}\right)\right|=o\left(B_{q}(x)\right)
$$

and that $D_{q}(x) \rightarrow \infty$, where

$$
D_{q}^{2}(x)=\sum_{k=0}^{\log _{q} x} \sigma_{k, q}^{2} \quad \text { and } \quad \sigma_{k, q}^{2}:=\frac{1}{q} \sum_{c \in E_{q}} f^{2}\left(c q^{k}\right)-m_{k, q}^{2} .
$$

Then, as $x \rightarrow \infty$,

$$
\frac{1}{x} \#\left\{n<x \mid \frac{f(n)-M_{q}(x)}{D_{q}(x)}<y\right\} \rightarrow \Phi(y),
$$

where $\Phi$ is the normal distribution function.

Similar distribution results for the sum-of-digits function of number systems related to substitution automata were considered by Dumont and Thomas [8]. For number systems whose bases satisfy linear recurrences we refer to $[6]$. 
Furthermore, Bassily and Kátai [1] studied the distribution of $q$-additive functions on polynomial sequences.

THEOREM 1. Let $f$ be a q-additive function such that $f\left(c q^{j}\right)=O(1)$ as $j \rightarrow \infty$ and $c \in E_{q}$. Assume that $D_{q}(x) /(\log x)^{\eta} \rightarrow \infty$ as $x \rightarrow \infty$ for some $\eta>0$ and let $P(x)$ be a polynomial with integer coefficients, degree $r$, and positive leading term. Then, as $x \rightarrow \infty$,

$$
\begin{aligned}
& \frac{1}{x} \#\left\{\begin{array}{l|l}
n<x & \frac{f(P(n))-M_{q}\left(x^{r}\right)}{D_{q}\left(x^{r}\right)}<y
\end{array}\right\} \rightarrow \Phi(y), \\
& \frac{1}{\pi(x)} \#\left\{p<x \mid \frac{f(P(p))-M_{q}\left(x^{r}\right)}{D_{q}\left(x^{r}\right)}<y\right\} \rightarrow \Phi(y) .
\end{aligned}
$$

This result relies on the fact that suitably modified centralized moments converge (cf. Lemma 4). Note also that this theorem was only stated (and proved) for $\eta=1 / 3$. However, a short inspection of the proof shows that $\eta>0$ is sufficient.

2. Joint distributions. It is a natural question to ask whether there are analogue results for the joint distribution of $q_{l}$-additive functions $f_{l}(n)$ (if $q_{1}, \ldots, q_{d}>1$ are pairwise coprime integers). For example, Hildebrand [14] announced that one always has

$$
\frac{1}{x} \#\left\{n<x \mid f_{l}(n)<y_{l}, 1 \leq l \leq d\right\} \rightarrow F_{1}\left(y_{1}\right) \ldots F_{d}\left(y_{d}\right)
$$

if $f_{l}$ satisfies (1.2) for all $l=1, \ldots, d$ and that there is a joint central limit theorem of the form

$$
\frac{1}{x} \#\left\{n<x \mid \frac{f_{l}(n)-M_{q_{l}}(x)}{D_{q_{l}}(x)}<y_{l}, 1 \leq l \leq d\right\} \rightarrow \Phi\left(y_{1}\right) \ldots \Phi\left(y_{d}\right)
$$

if $B_{q_{l}}(x) \rightarrow \infty$ and $B_{q_{l}}\left(x^{\eta}\right) \sim B_{q_{l}}(x)$ for every $\eta>0$ as $x \rightarrow \infty$. (Note that the sum-of-digits function $s_{q}(n)$ is not covered by this result.)

In this paper we will first extend the above result of Bassily and Kátai to the joint distribution of $q_{l}$-additive functions $f_{l}(1 \leq l \leq d)$ on specific polynomial sequences if $q_{1}, \ldots, q_{d}$ are pairwise coprime.

THEOREM 2. Let $q_{1}, \ldots, q_{d}>1$ be pairwise coprime integers and let $f_{l}$, $1 \leq l \leq d$, be $q_{l}$-additive functions such that $f_{l}\left(c q_{l}^{j}\right)=O(1)$ as $j \rightarrow \infty$ and $c \in E_{q_{l}}$. Assume that $D_{q_{l}}(x) /(\log x)^{\eta} \rightarrow \infty$ as $x \rightarrow \infty, 1 \leq l \leq d$, for some $\eta>0$ and let $P_{l}(x)$ be polynomials with integer coefficients of different degrees $r_{l}$ and positive leading terms, $1 \leq l \leq d$. Then, as $x \rightarrow \infty$,

$$
\begin{aligned}
& \frac{1}{x} \#\left\{n<x \mid \frac{f_{l}\left(P_{l}(n)\right)-M_{q_{l}}\left(x^{r_{l}}\right)}{D_{q_{l}}\left(x^{r_{l}}\right)}<y_{l}, 1 \leq l \leq d\right\} \rightarrow \Phi\left(y_{1}\right) \ldots \Phi\left(y_{d}\right), \\
& \frac{1}{\pi(x)} \#\left\{p<x \mid \frac{f_{l}\left(P_{l}(p)\right)-M_{q_{l}}\left(x^{r_{l}}\right)}{D_{q_{l}}\left(x^{r_{l}}\right)}<y_{l}, 1 \leq l \leq d\right\} \rightarrow \Phi\left(y_{1}\right) \ldots \Phi\left(y_{d}\right) .
\end{aligned}
$$


Corollary 1. Let $q_{1}, \ldots, q_{d}>1$ be pairwise coprime integers and let $P_{l}(x)$ be polynomials with integer coefficients of different degrees $r_{l}$ and positive leading terms, $1 \leq l \leq d$. Then, as $x \rightarrow \infty$,

$$
\begin{aligned}
& \frac{1}{x} \#\left\{n<x \mid \frac{s_{q_{l}}\left(P_{l}(n)\right)-\frac{q_{l}-1}{2} \log _{q_{l}} x^{r_{l}}}{\sqrt{\frac{q_{l}^{2}-1}{12} \log _{q_{l}} x^{r_{l}}}}<y_{l}, 1 \leq l \leq d\right\} \rightarrow \Phi\left(y_{1}\right) \ldots \Phi\left(y_{d}\right), \\
& \frac{1}{\pi(x)} \#\left\{p<x \mid \frac{s_{q_{l}}\left(P_{l}(p)\right)-\frac{q_{l}-1}{2} \log _{q_{l}} x^{r_{l}}}{\sqrt{\frac{q_{l}^{2}-1}{12} \log _{q_{l}} x^{r_{l}}}}<y_{l}, 1 \leq l \leq d\right\} \rightarrow \Phi\left(y_{1}\right) \ldots \Phi\left(y_{d}\right) .
\end{aligned}
$$

This theorem contains an unnatural condition, namely that one has to consider polynomials $P_{l}(x)$ with different degrees $r_{l}$. It would seem that this condition is not necessary. However, this is the crux of the matter. By using a variation of Bassily and Kátai's proof (combined with Baker's theorem on linear forms of logarithms) we could handle the case $d=2$ with linear polynomals $P_{l}(x)=A_{l} x+B_{l}$.

TheOREM 3. Let $q_{1}, q_{2}>1$ be coprime integers and let $f_{l}$ be $q_{l}$-additive functions such that $f_{l}\left(c q_{l}^{j}\right)=O(1)$ as $j \rightarrow \infty$ and $c \in E_{q_{l}}, l=1,2$. Assume that $D_{q_{l}}(x) /(\log x)^{\eta} \rightarrow \infty$ as $x \rightarrow \infty, l=1,2$, for some $\eta>0$. Let $P_{l}(x)=$ $A_{l} x+B_{l}, l=1,2$, be arbitrary linear polynomials with integer coefficients and positive leading terms $A_{l}$ coprime to $q_{l}$. Then, as $x \rightarrow \infty$,

$$
\frac{1}{x} \#\left\{n<x \mid \frac{f_{l}\left(P_{l}(n)\right)-M_{q_{l}}(x)}{D_{q_{l}}(x)}<y_{l}, l=1,2\right\} \rightarrow \Phi\left(y_{1}\right) \Phi\left(y_{2}\right) .
$$

Corollary 2. Let $q_{1}, q_{2}>1$ be coprime integers. Then, as $x \rightarrow \infty$,

$$
\frac{1}{x} \#\left\{\begin{array}{l|l|l|}
n<x & \frac{s_{q_{l}}(n)-\frac{q_{l}-1}{2} \log _{q_{l}} x}{\sqrt{\frac{q_{l}^{2}-1}{12} \log _{q_{l}} x}}<y_{l}, \quad l=1,2
\end{array}\right\} \rightarrow \Phi\left(y_{1}\right) \Phi\left(y_{2}\right) .
$$

Interestingly, there is even a local version of Corollary 2 .

TheOREM 4. Let $q_{1}, q_{2}>1$ be coprime integers and set $d=\operatorname{gcd}\left(q_{1}-1\right.$, $\left.q_{2}-1\right)$. Then, as $x \rightarrow \infty$,

$$
\begin{aligned}
\frac{1}{x} \#\{ & \left.n<x \mid s_{q_{1}}(n)=k_{1}, s_{q_{2}}(n)=k_{2}\right\} \\
& =d \prod_{l=1}^{2}\left(\frac{1}{\sqrt{2 \pi \frac{q_{l}^{2}-1}{12} \log _{q_{l}} x}} \exp \left(-\frac{\left(k_{l}-\frac{q_{l}-1}{2} \log _{q_{l}} x\right)^{2}}{2 \frac{q_{l}^{2}-1}{12} \log _{q_{l}} x}\right)\right)+o\left((\log x)^{-1}\right)
\end{aligned}
$$

uniformly for all integers $k_{1}, k_{2} \geq 0$ with $k_{1} \equiv k_{2} \bmod d$. 
Note that $s_{q_{l}}(n) \equiv n \bmod \left(q_{l}-1\right)$. Thus we always have $s_{q_{1}}(n) \equiv$ $s_{q_{2}}(n) \bmod d$ and consequently

$$
\#\left\{n<x \mid s_{q_{1}}(n)=k_{1}, s_{q_{2}}(n)=k_{2}\right\}=0
$$

if $k_{1} \not \equiv k_{2} \bmod d$.

There are some other results indicating that the $q_{l}$-ary digital expansions are asymptotically independent for different bases $q_{l}$; e.g. Kim [18] $\left({ }^{1}\right)$ showed that for all integers $c_{1}, \ldots, c_{d}$,

$$
\frac{1}{x}\left|\left\{n<x \mid s_{q_{j}}(n) \equiv c_{j} \bmod m_{j}(1 \leq j \leq d)\right\}\right|=\frac{1}{m_{1} \ldots m_{d}}+O\left(x^{-\delta}\right)
$$

with

$$
\delta=\frac{1}{120 d^{2} q^{2} m^{2}},
$$

where $q_{1}, \ldots, q_{d}>1$ are pairwise coprime integers and $m_{1}, \ldots, m_{d}$ are positive integers such that

$$
\operatorname{gcd}\left(q_{j}-1, m_{j}\right)=1 \quad(1 \leq j \leq d) ;
$$

$q=\max \left\{q_{1}, \ldots, q_{d}\right\}, m=\max \left\{m_{1}, \ldots, m_{d}\right\}$ and the $O$-constant depends only on $d$ and $q$. (This result sharpens a result by Bésineau [2] and solves a conjecture of Gelfond [11].)

Drmota and Larcher [7] used a variation of Kim's method to prove that a $d$-dimensional sequence $\left(\alpha_{1} s_{q_{1}}(n), \ldots, \alpha_{d} s_{q_{d}}(n)\right)_{n \geq 0}$ is uniformly distributed modulo 1 if and only if $\alpha_{1}, \ldots, \alpha_{d}$ are irrational. (Grabner, Liardet and Tichy [13] could prove a similar theorem by ergodic means.)

Another problem has been considered by Senge and Straus [26]. They proved that if $q_{1}$ and $q_{2}$ are coprime and $c$ is any given positive constant then there are only finitely many $n \geq 0$ such that

$$
s_{q_{1}}(n) \leq c \text { and } s_{q_{2}}(n) \leq c .
$$

This result was later generalized and sharpened by Stewart [28], Schlickewei $[22,23]$ and by Pethő and Tichy [21]. The proofs use Baker's method for linear forms of logarithms and the $p$-adic version of Schmidt's subspace theorem by Schlickewei applied to $S$-unit equations.

One would get a much deeper insight into all these results if one could prove a local version of Theorem 2, e.g. asymptotic expansions or general estimates for the numbers

$$
\frac{1}{x} \#\left\{n<x \mid s_{q}\left(n^{2}\right)=k\right\}
$$

or for

$$
\frac{1}{\pi(x)} \#\left\{p<x \mid s_{q}(p)=k\right\}
$$

$\left({ }^{1}\right)$ For brevity we restrict to the sum-of-digits function $s_{q}(n)$. 
(and of course multivariate versions). It seems that problems of this kind are extremely difficult, e.g. it is an open question whether there are infinitely many primes $p$ with even sum-of-digits function $s_{2}(p)$. The best known results concerning these questions are due to Fouvry and Mauduit $[9,10]$ who proved that

$$
\frac{1}{x} \#\left\{n<x \mid n \in \mathbb{P} \vee\left(n=n_{1} \cdot n_{2} \wedge n_{1}, n_{2} \in \mathbb{P}\right), s_{q}(n) \equiv 0 \bmod 2\right\} \geq c>0
$$

for some constant $c>0$. ( $\mathbb{P}$ denotes the set of primes.)

These questions are also related to two other conjectures of Gelfond [11], namely that $s_{q}(P(n))$ and $s_{q}(p)$ are uniformly distributed modulo $m$.

Remark. Schmidt [25] and Schmid [24] discussed the joint distribution of $s_{2}\left(k_{l} n\right)$ for different odd integers $k_{l}, 1 \leq l \leq d$. (The distribution modulo $m$ was investigated by Solinas [27].) It is surely possible to extend their result to the joint distribution of $f_{l}\left(P_{l}(n)\right), 1 \leq l \leq d$, where $f_{l}$ are $q_{l}$-additive functions, $P_{l}$ are (certain) integer polynomials, and $q_{l}>1$ arbitrary integers (e.g. all equal). However, we will not discuss this question here.

3. Proof of Theorem 2. As already mentioned, Theorem 2 is a direct generalization of Bassily and Kátai's result of [1]. Therefore we can proceed as in [1].

The first two lemmata on exponential sums are stated in [1]; a proof can also be found in [15].

Lemma 1. Let $f(y)$ be a polynomial of degree $k$ of the form

$$
f(y)=\frac{a}{b} y^{k}+\alpha_{1} y^{k-1}+\ldots+\alpha_{k}
$$

with $\operatorname{gcd}(a, b)=1$. Let $\tau$ be a positive number satisfying

$$
\tau \geq 2^{3(k-2)} \quad \text { and } \quad(\log x)^{\tau}<b<x^{k}(\log x)^{-\tau} .
$$

Then, as $x \rightarrow \infty$,

$$
\frac{1}{x} \sum_{n<x} e(f(n))=O\left((\log x)^{-\tau}\right) .
$$

Lemma 2. Let $f(y)$ be as in Lemma 1 and $\tau_{0}, \tau$ arbitrary positive numbers satisfying

$$
\tau \geq 2^{6 k} \tau_{0} \quad \text { and } \quad(\log x)^{\tau}<b<x^{k}(\log x)^{-\tau} .
$$

Then, as $x \rightarrow \infty$,

$$
\frac{1}{\pi(x)} \sum_{p<x} e(f(p))=O\left((\log x)^{-\tau_{0}}\right) .
$$

The third lemma is proved in [1] with the help of Lemmata 1 and 2 and the inequality of Erdős-Turán. 
Lemma 3. Let $0<\Delta<1$ and

$$
U_{b, q, \Delta}:=[0, \Delta] \cup \bigcup_{b=1}^{q-1}[b / q-\Delta, b / q+\Delta] \cup[1-\Delta, 1] .
$$

Suppose that $P(x)$ is an integer polynomial of degree $r$ with positive leading term. Then for every $\varepsilon>0$ and arbitrary $\lambda>0$ we have uniformly for $\left(\log _{q} x\right)^{\varepsilon}<j<r \log _{q} x-\left(\log _{q} x\right)^{\varepsilon}$ and $0<\Delta<1 /(2 q)$, as $x \rightarrow \infty$,

$$
\begin{gathered}
\frac{1}{x} \#\left\{n<x \mid\left\{\frac{P(n)}{q^{j+1}}\right\} \in U_{b, q, \Delta}\right\} \ll \Delta+(\log x)^{-\lambda}, \\
\frac{1}{\pi(x)} \#\left\{p<x \mid\left\{\frac{P(p)}{q^{j+1}}\right\} \in U_{b, q, \Delta}\right\} \ll \Delta+(\log x)^{-\lambda} .
\end{gathered}
$$

We will also make use of the following limiting relations for centralized moments of $q$-additive functions (see [1]).

LEMma 4. Let $f$ be a q-additive function such that $f\left(c q^{j}\right)=O(1)$ as $j \rightarrow \infty$ and $c \in E_{q}$ and let $P(x)$ be a polynomial with integer coefficients, degree $r$, and positive leading term. Furthermore, suppose that for some $\eta>0$ we have $D_{q}\left(x^{r}\right) /(\log x)^{\eta} \rightarrow \infty$ as $x \rightarrow \infty$. Define $f_{1}$ for $n<x^{r}$ by

$$
f_{1}(n)=\sum_{\left(\log _{q} x\right)^{\eta} \leq j \leq r \log _{q} x-\left(\log _{q} x\right)^{\eta}} f\left(a_{q, j}(n) q^{j}\right)
$$

and set

$$
\begin{aligned}
& M_{q, 1}\left(x^{r}\right):=\sum_{\left(\log _{q} x\right)^{\eta} \leq k \leq r \log _{q} x-\left(\log _{q} x\right)^{\eta}} m_{k, q}, \\
& D_{q, 1}^{2}\left(x^{r}\right):=\sum_{\left(\log _{q} x\right)^{\eta} \leq k \leq r \log _{q} x-\left(\log _{q} x\right)^{\eta}} \sigma_{k, q}^{2} .
\end{aligned}
$$

Then, as $x \rightarrow \infty$,

$$
\begin{gathered}
\frac{1}{x} \# \sum_{n<x}\left(\frac{f_{1}(P(n))-M_{q, 1}\left(x^{r}\right)}{D_{q, 1}\left(x^{r}\right)}\right)^{k} \rightarrow \int_{-\infty}^{\infty} z^{k} d \Phi(z), \\
\frac{1}{\pi(x)} \# \sum_{p<x}\left(\frac{f_{1}(P(p))-M_{q, 1}\left(x^{r}\right)}{D_{q, 1}\left(x^{r}\right)}\right)^{k} \rightarrow \int_{-\infty}^{\infty} z^{k} d \Phi(z) .
\end{gathered}
$$

In [1] this property is only proved for $\eta=1 / 3$. However, as already mentioned, it is also true for any $\eta>0$.

Proposition 1. Let $N_{l}=\left[\log _{q_{l}} x\right], 1 \leq l \leq d$, let $\lambda>0$ be an arbitrary constant and $h_{l}, 1 \leq l \leq d$, be positive integers. Furthermore, let $P_{l}(x), 1 \leq$ $l \leq d$, be integer polynomials with non-negative leading terms and different degrees $r_{l} \geq 1$. Then for integers 


$$
N_{l}^{\eta} \leq k_{1}^{(l)}<k_{2}^{(l)}<\ldots<k_{h_{l}}^{(l)} \leq r_{l} N_{l}-N_{l}^{\eta} \quad(1 \leq l \leq d)
$$

(with some $\eta>0$ ) we have, as $x \rightarrow \infty$,

$$
\begin{aligned}
\frac{1}{x} \#\left\{n<x \mid a_{q_{l}, k_{j}^{(l)}}\left(P_{l}(n)\right)=b_{j}^{(l)}, 0\right. & \left.\leq j \leq h_{l}, 1 \leq l \leq d\right\} \\
& =\frac{1}{q_{1}^{h_{1}} \ldots q_{d}^{h_{d}}}+O\left((\log x)^{-\lambda}\right)
\end{aligned}
$$

and

$$
\begin{aligned}
\frac{1}{\pi(x)} \#\left\{p<x \mid a_{q_{l}, k_{j}^{(l)}}\left(P_{l}(p)\right)=b_{j}^{(l)},\right. & \left.0 \leq j \leq h_{l}, 1 \leq l \leq d\right\} \\
& =\frac{1}{q_{1}^{h_{1}} \ldots q_{d}^{h_{d}}}+O\left((\log x)^{-\lambda}\right)
\end{aligned}
$$

uniformly for $b_{j}^{(l)} \in E_{q_{l}}$ and $k_{j}^{(l)}$ in the given range, where the implicit constant of the error term may depend on $q_{l}$, on the polynomials $P_{l}$, on $h_{l}$ and on $\lambda$.

Proof. We follow [1]. Let $f_{b, q, \Delta}(x)$ be defined by

$$
f_{b, q, \Delta}(x):=\frac{1}{\Delta} \int_{-\Delta / 2}^{\Delta / 2} \mathbf{1}_{[b / q,(b+1) / q]}(\{x+z\}) d z,
$$

where $\mathbf{1}_{A}$ is the characteristic function of the set $A$ and $\{x\}=x-[x]$ the fractional part of $x$. The Fourier coefficients of the Fourier series $f_{b, q, \Delta}(x)$ $=\sum_{m \in \mathbb{Z}} d_{m, b, q, \Delta} e(m x)$ are given by

$$
d_{0, b, q, \Delta}=1 / q
$$

and for $m \neq 0$ by

$$
d_{m, b, q, \Delta}=\frac{e(-m b / q)-e(-m(b+1) / q)}{2 \pi i m} \cdot \frac{e(m \Delta / 2)-e(-m \Delta / 2)}{2 \pi i m \Delta} .
$$

Note that $d_{m, b, q, \Delta}=0$ if $m \neq 0$ and $m \equiv 0 \bmod q$ and that

$$
\left|d_{m, b, q, \Delta}\right| \leq \min \left(\frac{1}{\pi|m|}, \frac{1}{\Delta \pi m^{2}}\right) .
$$

By definition we have

$$
0 \leq f_{b, q, \Delta}(x) \leq 1
$$

and

$$
f_{b, q, \Delta}(x)= \begin{cases}1 & \text { if } x \in[b / q+\Delta,(b+1) / q-\Delta], \\ 0 & \text { if } x \in[0,1] \backslash[b / q-\Delta,(b+1) / q+\Delta] .\end{cases}
$$

So if we set

$$
t\left(y_{1}, \ldots, y_{d}\right):=\prod_{l=1}^{d} \prod_{j=1}^{h_{l}} f_{b_{j}^{(l)}, q_{l}, \Delta}\left(\frac{y_{l}}{q_{l}^{k_{j}^{(l)}}+1}\right)
$$


then for $\Delta<1 /(2 q)$ we get

$$
\begin{aligned}
\mid \#\left\{n<x \mid a_{q_{l}, k_{j}^{(l)}}\left(P_{l}(n)\right)=b_{j}^{(l)}, 0 \leq j \leq h_{l}, 1 \leq l \leq d\right\} \\
\quad-\sum_{n<x} t\left(P_{1}(n), \ldots, P_{d}(n)\right) \mid \\
\leq \sum_{l=1}^{d} \sum_{j=1}^{h_{l}} \#\left\{n<x \mid\left\{\frac{P_{l}(n)}{q_{l}^{k_{j}^{(l)}}+1}\right\} \in U_{b_{j}^{(l)}, q_{l}, \Delta}\right\} \ll \Delta x+x(\log x)^{-\lambda}
\end{aligned}
$$

and

$$
\begin{aligned}
\mid \#\left\{p<x \mid a_{q_{l}, k_{j}^{(l)}}\left(P_{l}(p)\right)=b_{j}^{(l)}, 0 \leq j \leq h_{l}, 1 \leq l\right. & \leq d\} \\
& -\sum_{p<x} t\left(P_{1}(p), \ldots, P_{d}(p)\right) \mid \\
\leq \sum_{l=1}^{d} \sum_{j=1}^{h_{l}} \#\left\{n<x \mid\left\{\frac{P_{l}(p)}{q_{l}^{(l)}+1}\right\} \in U_{b_{j}^{(l)}, q_{l}, \Delta}\right\} & \ll \Delta \pi(x)+\pi(x)(\log x)^{-\lambda},
\end{aligned}
$$

where $U_{b_{j}^{(l)}, q_{l}, \Delta}$ is given in Lemma 3 .

For convenience, let $\mathbf{m}_{l}=\left(m_{1}^{(l)}, \ldots, m_{h_{l}}^{(l)}\right)$ denote $h_{l}$-dimensional integer vectors and $\mathbf{v}_{l}=\left(q_{l}^{-k_{1}^{(l)}-1}, \ldots, q_{l}^{-k_{h_{l}}^{(l)}-1}\right), 1 \leq l \leq d$. Furthermore set

$$
T_{\mathbf{m}_{1}, \ldots, \mathbf{m}_{d}}:=\prod_{l=1}^{d} \prod_{j=1}^{h_{l}} d_{m_{j}^{(l)}, b_{j}^{(l)}, q_{l}, \Delta} .
$$

Then $t\left(P_{1}(n), \ldots, P_{d}(n)\right)$ has Fourier series expansion

$$
t\left(y_{1}, \ldots, y_{d}\right)=\sum_{\mathbf{m}_{1}, \ldots, \mathbf{m}_{d}} T_{\mathbf{m}_{1}, \ldots, \mathbf{m}_{d}} e\left(\mathbf{m}_{1} \cdot \mathbf{v}_{1} y_{1}+\ldots+\mathbf{m}_{d} \cdot \mathbf{v}_{d} y_{d}\right) .
$$

Thus, we are led to consider the exponential sums

$$
\begin{aligned}
& S_{1}=\sum_{\mathbf{m}_{1}, \ldots, \mathbf{m}_{d}} T_{\mathbf{m}_{1}, \ldots, \mathbf{m}_{d}} \sum_{n<x} e\left(\mathbf{m}_{1} \cdot \mathbf{v}_{1} P_{1}(n)+\ldots+\mathbf{m}_{d} \cdot \mathbf{v}_{d} P_{d}(n)\right), \\
& S_{2}=\sum_{\mathbf{m}_{1}, \ldots, \mathbf{m}_{d}} T_{\mathbf{m}_{1}, \ldots, \mathbf{m}_{d}} \sum_{p<x} e\left(\mathbf{m}_{1} \cdot \mathbf{v}_{1} P_{1}(p)+\ldots+\mathbf{m}_{d} \cdot \mathbf{v}_{d} P_{d}(p)\right) .
\end{aligned}
$$

Let us consider for a moment just the first sum $S_{1}$. If $\mathbf{m}_{1}, \ldots, \mathbf{m}_{d}$ are all zero then

$$
T_{\mathbf{m}_{1}, \ldots, \mathbf{m}_{d}} \sum_{n<x} e\left(\mathbf{m}_{1} \cdot \mathbf{v}_{1} P_{1}(n)+\ldots+\mathbf{m}_{d} \cdot \mathbf{v}_{d} P_{d}(n)\right)=\frac{x+O(1)}{q_{1}^{h_{1}} \ldots q_{d}^{h_{d}}},
$$

which provides the leading term. Furthermore, if there exist $l$ and $j$ with $m_{j}^{(l)} \neq 0$ and $m_{j}^{(l)} \equiv 0 \bmod q_{l}$ then $T_{\mathbf{m}_{1}, \ldots, \mathbf{m}_{d}}=0$. So it remains to consider 
the case where there exist $l$ and $j$ with $m_{j}^{(l)} \not \equiv 0 \bmod q_{l}$. Here the exponent is of the form

$$
\mathbf{m}_{1} \cdot \mathbf{v}_{1} P_{1}(n)+\ldots+\mathbf{m}_{d} \cdot \mathbf{v}_{d} P_{d}(n)=\frac{a_{1}}{b_{1}} P_{1}(n)+\ldots+\frac{a_{d}}{b_{d}} P_{d}(n)
$$

in which we assume that $\operatorname{gcd}\left(a_{l}, b_{l}\right)=1,1 \leq l \leq d$. The first observation is that for any $l$ for which there exists $j$ with $m_{j}^{(l)} \not \equiv 0 \bmod q_{l}$ there exists $\eta_{l}>0$ (only depending on $q_{l}$ ) such that $b_{l} \geq q_{l}^{\eta_{l} k_{s}^{(l)}}$ if $m_{s}^{(l)} \neq 0, m_{s}^{(l)} \not \equiv$ $0 \bmod q_{l}$ and $m_{s+1}^{(l)}=m_{s+2}^{(l)}=\ldots=m_{h_{l}}^{(l)}=0$ (cf. [1]). For the reader's convenience we repeat the argument. Suppose that the prime factorization of $q_{l}$ is given by $q_{l}=p_{1}^{e_{1}} \ldots p_{k}^{e_{k}}$. If $m_{s}^{(l)} \not \equiv 0 \bmod q_{l}$ then there exists $t$ such that $m_{s}^{(l)} \not \equiv 0 \bmod p_{t}^{e_{t}}$. Now we have

$$
b_{l}\left(m_{s}^{(l)}+q_{l}^{k_{s}^{(l)}-k_{s-1}^{(l)}} m_{s-1}^{(l)}+\ldots+q_{l}^{k_{s}^{(l)}-k_{1}^{(l)}} m_{1}^{(l)}\right)=a_{l} q_{l}^{k_{s}^{(l)}+1} .
$$

Hence $b_{l} \equiv 0 \bmod p_{t}^{k_{s}^{(l)}} e_{t}$ and consequently $b_{l} \geq p_{t}^{k_{s}^{(l)} e_{t}} \geq q_{l}^{\eta_{l} k_{s}^{(l)}}$. Note that we also have $b_{l} \leq q_{l}^{\eta_{l} k_{h_{l}}^{(l)}}$.

Now let $D$ denote the set of $l \in\{1, \ldots, d\}$ such that there exists $j$ with $m_{j}^{(l)} \not \equiv 0 \bmod q_{l}$. Since all degrees $r_{l}$ are different there exists a unique $l_{0}$ with $r_{l_{0}}=\max \left\{r_{l} \mid l \in D\right\}$. We now want to apply Lemma 1 with $k=r_{l_{0}}$ and $b=b_{l_{0}}$. If $k_{j}^{(l)}$ are in the range (3.1) then for every $\tau>0$ there exists $x_{0}(\tau)$ such that for $x \geq x_{0}(\tau)$,

$$
(\log x)^{\tau}<b_{l_{0}}<x^{r_{l_{0}}}(\log x)^{-\tau} .
$$

Consequently, we can apply Lemma 1 to obtain

$$
\begin{aligned}
\frac{1}{x} \#\{n<x & \left.\mid a_{q_{l}, k_{j}^{(l)}}(P(n))=b_{j}^{(l)}, 0 \leq j \leq h_{l}, 1 \leq l \leq d\right\} \\
& =\frac{1}{q_{1}^{h_{1}} \ldots q_{d}^{h_{d}}}+O\left((\log x)^{-\lambda} \sum_{\mathbf{m} \neq \mathbf{0}}\left|T_{\mathbf{m}_{1}, \ldots, \mathbf{m}_{d}}\right|\right)+O\left(\Delta+(\log x)^{-\lambda}\right),
\end{aligned}
$$

where $\mathbf{m}=\left(\mathbf{m}_{1}, \ldots, \mathbf{m}_{d}\right)$. Since

$$
\sum_{\mathbf{m} \neq \mathbf{0}}\left|T_{\mathbf{m}_{1}, \ldots, \mathbf{m}_{d}}\right| \leq(2+2 \log (1 / \Delta))^{h_{1}+\ldots+h_{d}}
$$

it is possible to choose $\Delta=(\log x)^{-\lambda_{1}}$ for a sufficiently large constant $\lambda_{1}$ such that (3.2) holds.

The proof of (3.3) runs along the same lines.

Corollary 3. Let $N_{l}=\left[\log _{q_{l}} x\right], 1 \leq l \leq d$, and $\lambda, \eta>0$. Then for integers $k_{j}^{(l)}$ satisfying

$$
N_{l}^{\eta} \leq k_{j}^{(l)}<r_{l} N_{l}-N_{l}^{\eta} \quad\left(1 \leq j \leq h_{l}, 1 \leq l \leq d\right)
$$


and $b_{j}^{(l)} \in E_{q_{l}}$, we uniformly have, as $x \rightarrow \infty$,

$$
\begin{aligned}
& \frac{1}{x} \#\left\{n<x \mid a_{q_{l}, k_{j}^{(l)}}\left(P_{l}(n)\right)=b_{j}^{(l)}, 0 \leq j \leq h_{l}, 1 \leq l \leq d\right\} \\
& \quad=\prod_{l=1}^{d}\left(\frac{1}{x} \#\left\{n<x \mid a_{q_{l}, k_{j}^{(l)}}\left(P_{l}(n)\right)=b_{j}^{(l)}, 0 \leq j \leq h_{l}\right\}\right)+O\left((\log x)^{-\lambda}\right)
\end{aligned}
$$

and

$$
\begin{aligned}
& \frac{1}{\pi(x)} \#\left\{p<x \mid a_{q_{l}, k_{j}^{(l)}}\left(P_{l}(p)\right)=b_{j}^{(l)}, 0 \leq j \leq h_{l}, 1 \leq l \leq d\right\} \\
& \quad=\prod_{l=1}^{d}\left(\frac{1}{\pi(x)} \#\left\{p<x \mid a_{q_{l}, k_{j}^{(l)}}\left(P_{l}(p)\right)=b_{j}^{(l)}, 0 \leq j \leq h_{l}\right\}\right)+O\left((\log x)^{-\lambda}\right) .
\end{aligned}
$$

Proof. If there exist $l$ and $j_{1}, j_{2}$ with $k_{j_{1}}^{(l)}=k_{j_{2}}^{(l)}$ but $b_{j_{1}}^{(l)} \neq b_{j_{2}}^{(l)}$ then both sides are zero.

So it remains to consider the case where for every $l$ the integers $k_{j}^{(l)}$, $1 \leq j \leq h_{l}$, are different, and without loss of generality we can assume that they are increasing. Hence we can directly apply Proposition 1.

Corollary 4. For any choice of integers $k_{l}, 1 \leq l \leq d$, we have, as $x \rightarrow \infty$,

$$
\begin{aligned}
\frac{1}{x} \sum_{n<x} \prod_{l=1}^{d}\left(\frac{f_{l, 1}\left(P_{l}(n)\right)-M_{q_{l}, 1}\left(x^{r_{l}}\right)}{D_{q_{l}, 1}\left(x^{r_{l}}\right)}\right)^{k_{l}} \\
\quad-\prod_{l=1}^{d}\left(\frac{1}{x} \sum_{n<x}\left(\frac{f_{l, 1}\left(P_{l}(n)\right)-M_{q_{l}, 1}\left(x^{r_{l}}\right)}{D_{q_{l}, 1}\left(x^{r_{l}}\right)}\right)^{k_{l}}\right) \rightarrow 0
\end{aligned}
$$

and

$$
\begin{aligned}
\frac{1}{\pi(x)} \sum_{p<x} & \prod_{l=1}^{d}\left(\frac{f_{l, 1}\left(P_{l}(p)\right)-M_{q_{l}, 1}\left(x^{r_{l}}\right)}{D_{q_{l}, 1}\left(x^{r_{l}}\right)}\right)^{k_{l}} \\
& \quad-\prod_{l=1}^{d}\left(\frac{1}{\pi(x)} \sum_{p<x}\left(\frac{f_{l, 1}\left(P_{l}(p)\right)-M_{q_{l}, 1}\left(x^{r_{l}}\right)}{D_{q_{l}, 1}\left(x^{r_{l}}\right)}\right)^{k_{l}}\right)
\end{aligned}
$$

Proof. In order to demonstrate how this property can be derived, we consider the case $d=2$ and $k_{1}=k_{2}=2$. Set $A_{l}=\left[\left(\log _{q_{l}} x\right)^{\eta}\right]$ and $B_{l}=$ $\left[\log _{q_{l}} x-\left(\log _{q_{l}} x\right)^{\eta}\right]$ and observe that

$$
f_{l, 1}\left(P_{l}(n)\right)-M_{q_{l}, 1}\left(x^{r_{l}}\right)=\sum_{j=A_{l}}^{B_{l}} \sum_{b \in E_{q_{l}}}\left(f_{l}\left(b q_{l}^{j}\right) \delta\left(a_{q_{l}, j}\left(P_{l}(n)\right), b\right)-\frac{m_{j, q_{l}}}{q_{l}}\right),
$$


where $\delta(x, y)$ denotes the Kronecker delta. Hence we have

$$
\begin{aligned}
\frac{1}{x} \sum_{n<x} & \left(\frac{f_{1,1}\left(P_{1}(n)\right)-M_{q_{1}, 1}\left(x^{r_{1}}\right)}{D_{q_{1}, 1}\left(x^{r_{1}}\right)}\right)^{2}\left(\frac{f_{2,1}\left(P_{2}(n)\right)-M_{q_{2}, 1}\left(x^{r_{2}}\right)}{D_{q_{2}, 1}\left(x^{r_{2}}\right)}\right)^{2} \\
= & \sum_{j_{1}=A_{1}}^{B_{1}} \sum_{j_{2}=A_{1}}^{B_{1}} \sum_{j_{3}=A_{2}}^{B_{2}} \sum_{j_{4}=A_{2}}^{B_{2}} \sum_{b_{1} \in E_{q_{1}}} \sum_{b_{2} \in E_{q_{1}}} \sum_{b_{3} \in E_{q_{2}}} \sum_{b_{4} \in E_{q_{2}}} \frac{1}{D_{q_{1}, 1}^{2}\left(x^{r_{1}}\right) D_{q_{2}, 1}^{2}\left(x^{r_{2}}\right)} \\
& \times \frac{1}{x} \sum_{n<x}\left(f_{1}\left(b_{1} q_{1}^{j_{1}}\right) \delta\left(a_{q_{1}, j_{1}}\left(P_{1}(n)\right), b_{1}\right)-\frac{m_{j_{1}, q_{1}}}{q_{1}}\right) \\
& \times\left(f_{1}\left(b_{2} q_{1}^{j_{2}}\right) \delta\left(a_{q_{1}, j_{2}}\left(P_{1}(n)\right), b_{2}\right)-\frac{m_{j_{2}, q_{1}}}{q_{1}}\right) \\
& \times\left(f_{2}\left(b_{3} q_{2}^{j_{3}}\right) \delta\left(a_{q_{2}, j_{3}}\left(P_{2}(n)\right), b_{3}\right)-\frac{m_{j_{3}, q_{2}}}{q_{2}}\right) \\
& \times\left(f_{2}\left(b_{4} q_{2}^{j_{4}}\right) \delta\left(a_{q_{2}, j_{4}}\left(P_{2}(n)\right), b_{4}\right)-\frac{m_{j_{4}, q_{2}}}{q_{2}}\right) .
\end{aligned}
$$

By Corollary 3 it follows that

$$
\begin{aligned}
& \frac{1}{x} \sum_{n<x}\left(f_{1}\left(b_{1} q_{1}^{j_{1}}\right) \delta\left(a_{q_{1}, j_{1}}\left(P_{1}(n)\right), b_{1}\right)-\frac{m_{j_{1}, q_{1}}}{q_{1}}\right) \\
& \quad\left(f_{1}\left(b_{2} q_{1}^{j_{2}}\right) \delta\left(a_{q_{1}, j_{2}}\left(P_{1}(n)\right), b_{2}\right)-\frac{m_{j_{2}, q_{1}}}{q_{1}}\right) \\
& \times\left(f_{2}\left(b_{3} q_{2}^{j_{3}}\right) \delta\left(a_{q_{2}, j_{3}}\left(P_{2}(n)\right), b_{3}\right)-\frac{m_{j_{3}, q_{2}}}{q_{2}}\right) \\
& \times\left(f_{2}\left(b_{4} q_{2}^{j_{4}}\right) \delta\left(a_{q_{2}, j_{4}}\left(P_{2}(n)\right), b_{4}\right)-\frac{m_{j_{4}, q_{2}}}{q_{2}}\right) \\
&= f_{1}\left(b_{1} q_{1}^{j_{1}}\right) f_{1}\left(b_{2} q_{1}^{j_{2}}\right) f_{2}\left(b_{3} q_{2}^{j_{3}}\right) f_{2}\left(b_{4} q_{2}^{j_{4}}\right) \\
& \quad \times \frac{1}{x} \#\left\{n<x \mid a_{q_{1}, j_{1}}\left(P_{1}(n)\right)=b_{1}, a_{q_{1}, j_{2}}\left(P_{1}(n)\right)=b_{2},\right. \\
&\left.\quad-a_{q_{2}, j_{3}}\left(P_{2}(n)\right)=b_{3}, a_{q_{2}, j_{4}}\left(P_{2}(n)\right)=b_{4}\right\} \\
& \quad \times \frac{1}{x} \#\left\{n<x \mid b_{1} q_{1}^{j_{1}}\right) f_{1}\left(b_{2} q_{1}^{j_{2}}\right) f_{2}\left(b_{3} q_{2}^{j_{3}}\right) \\
& \quad \times \frac{m_{j_{4}, q_{2}}}{q_{2}} \mp \ldots+\frac{m_{j_{1}, q_{1}}}{q_{1}} \cdot \frac{m_{j_{2}, q_{1}}}{q_{1}} \cdot \frac{m_{j_{3}, q_{2}}}{q_{2}} \cdot \frac{m_{j_{4}, q_{2}}}{q_{2}}
\end{aligned}
$$




$$
\begin{aligned}
& =\left(f_{1}\left(b_{1} q_{1}^{j_{1}}\right) f_{1}\left(b_{2} q_{1}^{j_{2}}\right) \frac{1}{x} \#\left\{n<x \mid a_{q_{1}, j_{1}}\left(P_{1}(n)\right)=b_{1}, a_{q_{1}, j_{2}}\left(P_{1}(n)\right)=b_{2}\right\}\right) \\
& \times\left(f_{2}\left(b_{3} q_{2}^{j_{3}}\right) f_{2}\left(b_{4} q_{2}^{j_{4}}\right)\right. \\
& \left.\times \frac{1}{x} \#\left\{n<x \mid a_{q_{2}, j_{3}}\left(P_{2}(n)\right)=b_{3}, a_{q_{2}, j_{4}}\left(P_{2}(n)\right)=b_{4}\right\}\right) \\
& -\left(f_{1}\left(b_{1} q_{1}^{j_{1}}\right) f_{1}\left(b_{2} q_{1}^{j_{2}}\right)\right. \\
& \left.\times \frac{1}{x} \#\left\{n<x \mid a_{q_{1}, j_{1}}\left(P_{1}(n)\right)=b_{1}, a_{q_{1}, j_{2}}\left(P_{1}(n)\right)=b_{2}\right\}\right) \\
& \times\left(f_{2}\left(b_{3} q_{2}^{j_{3}}\right) \frac{1}{x} \#\left\{n<x \mid a_{q_{2}, j_{3}}\left(P_{2}(n)\right)=b_{3}\right\}\right) \frac{m_{j_{4}, q_{2}}}{q_{2}} \\
& \mp \ldots+\left(\frac{m_{j_{1}, q_{1}}}{q_{1}} \cdot \frac{m_{j_{2}, q_{1}}}{q_{1}}\right)\left(\frac{m_{j_{3}, q_{2}}}{q_{2}} \cdot \frac{m_{j_{4}, q_{2}}}{q_{2}}\right)+O\left((\log x)^{-\lambda}\right) \\
& =\left(\frac{1}{x} \sum_{n<x}\left(f_{1}\left(b_{1} q_{1}^{j_{1}}\right) \delta\left(a_{q_{1}, j_{1}}\left(P_{1}(n)\right), b_{1}\right)-\frac{m_{j_{1}, q_{1}}}{q_{1}}\right)\right. \\
& \left.\times\left(f_{1}\left(b_{2} q_{1}^{j_{2}}\right) \delta\left(a_{q_{1}, j_{2}}\left(P_{1}(n)\right), b_{2}\right)-\frac{m_{j_{2}, q_{1}}}{q_{1}}\right)\right) \\
& \times\left(\frac{1}{x} \sum_{n<x}\left(f_{2}\left(b_{3} q_{2}^{j_{3}}\right) \delta\left(a_{q_{2}, j_{3}}\left(P_{2}(n)\right), b_{3}\right)-\frac{m_{j_{3}, q_{2}}}{q_{2}}\right)\right. \\
& \left.\times\left(f_{2}\left(b_{4} q_{2}^{j_{4}}\right) \delta\left(a_{q_{2}, j_{4}}\left(P_{2}(n)\right), b_{4}\right)-\frac{m_{j_{4}, q_{2}}}{q_{2}}\right)\right) \\
& +O\left((\log x)^{-\lambda}\right) \text {. }
\end{aligned}
$$

So we directly obtain the claimed result with an error term of the form $O\left((\log x)^{-\lambda+4-4 \eta}\right)$.

By combining Lemma 4, Corollary 4, and the Fréchet-Shohat theorem it follows that, as $x \rightarrow \infty$,

$$
\begin{gathered}
\frac{1}{x} \#\left\{n<x \mid \frac{f_{l, 1}\left(P_{l}(n)\right)-M_{q_{l}, 1}\left(x^{r_{l}}\right)}{D_{q_{l}, 1}\left(x^{r_{l}}\right)}<y_{l}, 1 \leq l \leq d\right\} \rightarrow \Phi\left(y_{1}\right) \ldots \Phi\left(y_{d}\right), \\
\frac{1}{\pi(x)} \#\left\{p<x \mid \frac{f_{l, 1}\left(P_{l}(p)\right)-M_{q_{l}, 1}\left(x^{r_{l}}\right)}{D_{q_{l}, 1}\left(x^{r_{l}}\right)}<y_{l}, 1 \leq l \leq d\right\} \rightarrow \Phi\left(y_{1}\right) \ldots \Phi\left(y_{d}\right) .
\end{gathered}
$$

Since

$$
\begin{aligned}
M_{q_{l}}\left(x^{r_{l}}\right)-M_{q_{l}, 1}\left(x^{r_{l}}\right) & =O\left((\log x)^{\eta}\right), \\
D_{q_{l}}\left(x^{r_{l}}\right)-D_{q_{l}, 1}\left(x^{r_{l}}\right) & =O\left((\log x)^{\eta}\right),
\end{aligned}
$$


it also follows that

$$
\max _{n<x}\left|\frac{f_{l}\left(P_{l}(n)\right)-M_{q_{l}}\left(x^{r_{l}}\right)}{D_{q_{l}}\left(x^{r_{l}}\right)}-\frac{f_{l, 1}\left(P_{l}(n)\right)-M_{q_{l}, 1}\left(x^{r_{l}}\right)}{D_{q_{l}, 1}\left(x^{r_{l}}\right)}\right| \rightarrow 0
$$

as $x \rightarrow \infty$. Consequently we finally obtain the limiting relations stated in Theorem 2 .

4. Proof of Theorem 3. The proof of Theorem 3 is similar to that of Theorem 2, i.e., we will prove an analogue to Proposition 1. However, the proof requires an additional ingredient, namely a proper version of Baker's theorem on linear forms. More precisely, we will use the following version due to Waldschmidt [29].

Lemma 5. Let $\alpha_{1}, \ldots, \alpha_{n}$ be non-zero algebraic numbers and $b_{1}, \ldots, b_{n}$ integers such that

$$
\alpha_{1}^{b_{1}} \ldots \alpha_{n}^{b_{n}} \neq 1
$$

and let $A_{1}, \ldots, A_{n} \geq e$ be real numbers with $\log A_{j} \geq h\left(\alpha_{j}\right)$, where $h(\cdot)$ denotes the absolute logarithmic height. Set $d=\left[\mathbb{Q}\left(\alpha_{1}, \ldots, \alpha_{n}\right): \mathbb{Q}\right]$. Then

$$
\left|\alpha_{1}^{b_{1}} \ldots \alpha_{n}^{b_{n}}-1\right| \geq \exp (-U)
$$

where

$$
\begin{aligned}
U & =2^{6 n+32} n^{3 n+6} d^{n+2}(1+\log d)(\log B+\log d) \log A_{1} \ldots \log A_{n}, \\
B & =\max \left\{2,\left|b_{1}\right|, \ldots,\left|b_{n}\right|\right\} .
\end{aligned}
$$

COROllary 5. Let $q_{1}, q_{2}>1$ be coprime integers and $m_{1}, m_{2}$ integers such that $m_{1} \not \equiv 0 \bmod q_{1}$ and $m_{2} \not \equiv 0 \bmod q_{2}$. Then there exists a constant $C>0$ such that for all integers $k_{1}, k_{2}>1$,

$$
\begin{aligned}
\left|\frac{m_{1}}{q_{1}^{k_{1}}}+\frac{m_{2}}{q_{2}^{k_{2}}}\right| & \\
& \geq \max \left(\frac{\left|m_{1}\right|}{q_{1}^{k_{1}}}, \frac{\left|m_{2}\right|}{q_{2}^{k_{2}}}\right) \cdot e^{-C \log q_{1} \log q_{2} \log \left(\max \left(k_{1}, k_{2}\right)\right) \cdot \log \left(\max \left(\left|m_{1}\right|,\left|m_{2}\right|\right)\right) .}
\end{aligned}
$$

Proof. Since $q_{1}, q_{2}>1$ are coprime integers and $m_{1} \not \equiv 0 \bmod q_{1}, m_{2} \not \equiv$ $0 \bmod q_{2}$ we surely have $m_{1} q_{1}^{-k_{1}}+m_{2} q_{2}^{-k_{2}} \neq 0$. So we can apply Lemma 5 for $n=3, \alpha_{1}=q_{1}, \alpha_{2}=q_{2}, \alpha_{3}=-m_{2} / m_{1}, b_{1}=k_{1}, b_{2}=-k_{2}, b_{3}=1$ and directly obtain

$$
\begin{aligned}
\left|\frac{m_{1}}{q_{1}^{k_{1}}}+\frac{m_{2}}{q_{2}^{k_{2}}}\right| & =\left|m_{1}\right| \cdot q_{1}^{k_{1}} \cdot\left|-q_{1}^{k_{1}} q_{2}^{-k_{2}} \frac{m_{2}}{m_{1}}-1\right| \\
& \geq\left|m_{1}\right| q_{1}^{k_{1}} e^{-C \log q_{1} \log q_{2} \log \left(\max \left(k_{1}, k_{2}\right)\right) \cdot \log \max \left(\left|m_{1}\right|,\left|m_{2}\right|\right) .}
\end{aligned}
$$

Since the problem is symmetric it is no loss of generality to assume that $\left|m_{1}\right| q_{1}^{-k_{1}} \geq\left|m_{2}\right| q_{2}^{-k_{2}}$. 
Finally we will use the following (trivial) lemma on exponential sums.

Lemma 6. Let $\alpha$ be a real number with $0<|\alpha| \leq 1 / 2$. Then, as $x \rightarrow \infty$,

$$
\sum_{n<x} e(\alpha n) \ll \frac{1}{|\alpha|} .
$$

Proposition 2. Let $P_{l}(x)=A_{l} x+B_{l}, l=1,2$, be linear polynomials with integer coefficients and non-negative leading terms $A_{l}$ which are coprime to $q_{l}$. Set $N_{l}=\left[\log _{q_{l}} x\right], l=1,2$, let $\lambda, \eta>0$ be arbitrary constants and let $h_{1}, h_{2}$ be positive integers. Then for integers

$$
N_{l}^{\eta} \leq k_{1}^{(l)}<k_{2}^{(l)}<\ldots<k_{h_{l}}^{(l)} \leq N_{l}-N_{l}^{\eta} \quad(l=1,2)
$$

we have, as $x \rightarrow \infty$,

$$
\begin{aligned}
\frac{1}{x} \#\left\{n<x \mid a_{q_{l}, k_{j}^{(l)}}\left(A_{l} n+B_{l}\right)=b_{j}^{(l)}, 0\right. & \left.\leq j \leq h_{l}, \quad l=1,2\right\} \\
& =\frac{1}{q_{1}^{h_{1}} q_{2}^{h_{2}}}+O\left((\log x)^{-\lambda}\right)
\end{aligned}
$$

uniformly for $b_{j}^{(l)} \in E_{q_{l}}$ and $k_{j}^{(l)}$ in the given range, where the implicit constant of the error term may depend on $q_{l}, h_{l}$ and $\lambda$.

Proof. The proof runs along the same lines as the proof of Proposition 1. The only problem is to estimate the sum

$$
\sum_{\left(\mathbf{m}_{1}, \mathbf{m}_{2}\right) \neq \mathbf{0}}\left|T_{\mathbf{m}_{1}, \mathbf{m}_{2}}\right| \cdot\left|\frac{1}{x} \sum_{n<x} e\left(\left(A_{1} \mathbf{m}_{1} \cdot \mathbf{v}_{1}+A_{2} \mathbf{m}_{2} \cdot \mathbf{v}_{2}\right) n\right)\right|,
$$

where $\mathbf{m}_{l}=\left(m_{1}^{(l)}, \ldots, m_{h_{l}}^{(l)}\right)$ and $\mathbf{v}_{l}=\left(q_{l}^{-k_{1}^{(l)}-1}, \ldots, q_{l}^{-k_{h_{l}}^{(l)}-1}\right), l=1,2$, such that the integers $k_{j}^{(l)}$ are in the given range (4.1).

First we fix $\Delta=(\log x)^{-\lambda_{0}}$ with an arbitrary (but fixed) constant $\lambda_{0}>0$. Furthermore, since

$$
\sum_{\exists l \exists j:\left|m_{j}^{(l)}\right|>(\log x)^{2 \lambda_{0}}}\left|T_{\mathbf{m}_{1}, \mathbf{m}_{2}}\right| \ll(\log x)^{-\lambda_{0}}
$$

we can restrict to those $\mathbf{m} \neq \mathbf{0}$ for which $\left|m_{j}^{(l)}\right| \leq(\log x)^{2 \lambda_{0}}$ for all $l, j$ and $m_{j}^{(l)} \not \equiv 0 \bmod q_{l}$ if $m_{j}^{(l)} \neq 0$.

We also note that it is also sufficient to consider just the case where $m_{j}^{(l)} \neq 0$ for all $j$ and $l=1,2$. (Otherwise we just reduce $h_{1}$ resp. $h_{2}$ to a smaller value and use the same arguments.)

Set $\delta=\eta /\left(h_{1}+h_{2}-1\right)$. Then there exists an integer $k$ with $0 \leq k \leq$ $h_{1}+h_{2}-2$ such that for all $j$ and $l=1,2$

$$
k_{j+1}^{(l)}-k_{j}^{(l)} \notin\left[(\log x)^{k \delta},(\log x)^{(k+1) \delta}\right) .
$$


So fix $k$ with this property. Before discussing the general case, let us consider two extremal ones.

First suppose that

$$
k_{j+1}^{(l)}-k_{j}^{(l)}<(\log x)^{k \delta}
$$

for all $j$ and $l=1,2$. Set

$$
\bar{m}_{l}=A_{l} \sum_{j=1}^{h_{l}} m_{j}^{(l)} q_{l}^{k_{h_{l}}^{(l)}-k_{j}^{(l)}} \quad(l=1,2) .
$$

Then we have $\bar{m}_{l} \not \equiv 0 \bmod q_{l}$ and $\log \left|\bar{m}_{l}\right| \ll(\log x)^{k \delta}$. Hence, we can apply Corollary 5 to

$$
A_{1} \mathbf{m}_{1} \cdot \mathbf{v}_{1}+A_{2} \mathbf{m}_{2} \cdot \mathbf{v}_{2}=\frac{\bar{m}_{1}}{q_{1}^{k_{h_{1}}^{(1)}+1}}+\frac{\bar{m}_{2}}{q_{2}^{k_{h_{2}}^{(1)}+1}}
$$

and obtain

$$
\left|A_{1} \mathbf{m}_{1} \cdot \mathbf{v}_{1}+A_{2} \mathbf{m}_{2} \cdot \mathbf{v}_{2}\right| \geq \max \left(q_{1}^{-k_{h_{1}}^{(1)}-1}, q_{2}^{-k_{h_{2}}^{(1)}-1}\right) e^{-C \log \log x(\log x)^{k \delta}}
$$

for some constant $C>0$. Since $\left|A_{1} \mathbf{m}_{1} \cdot \mathbf{v}_{1}+A_{2} \mathbf{m}_{2} \cdot \mathbf{v}_{2}\right| \leq 1 / 2$, from Lemma 6 we get

$$
\begin{aligned}
\left|\frac{1}{x} \sum_{n<x} e\left(\left(A_{1} \mathbf{m}_{1} \cdot \mathbf{v}_{1}+A_{2} \mathbf{m}_{2} \cdot \mathbf{v}_{2}\right) n\right)\right| \\
\ll \frac{1}{x} q^{\log _{q} x-(\log x)^{\left(h_{1}+h_{2}-1\right) \delta}} e^{C \log \log x(\log x)^{k \delta}} \\
=e^{-(\log x)^{\left(h_{1}+h_{2}-1\right) \delta} / \log q+C \log \log x(\log x)^{k \delta}} \ll(\log x)^{-\lambda}
\end{aligned}
$$

for any given $\lambda>0$.

Next suppose that

$$
k_{j+1}^{(l)}-k_{j}^{(l)} \geq(\log x)^{(k+1) \delta}
$$

for all $j$ and $l=1,2$. Here we set $\bar{m}_{l}=A_{l} m_{1}^{(l)}(l=1,2)$ and obtain

$$
\begin{aligned}
\mid A_{1} \mathbf{m}_{1} \cdot \mathbf{v}_{1}+ & A_{2} \mathbf{m}_{2} \cdot \mathbf{v}_{2} \mid \\
\geq & \left|\frac{\bar{m}_{1}}{q_{1}^{k_{1}^{(1)}}+1}+\frac{\bar{m}_{2}}{q_{2}^{k_{1}^{(2)}}+1}\right|-\left|\sum_{j_{1}=2}^{h_{1}} \frac{m_{j_{1}}^{(1)}}{q_{1}^{(1)}+1}\right|-\left|\sum_{j_{2}=2}^{h_{2}} \frac{m_{j_{2}}^{(2)}}{k_{2}^{(2)}+1}\right| \\
\geq & \max \left(q_{1}^{-k_{h_{1}}^{(1)}-1}, q_{2}^{-k_{h_{2}}^{(1)}-1}\right) e^{-C(\log \log x)^{2}} \\
& -O\left((\log x)^{2 \lambda_{0}} \max \left(q_{1}^{-k_{h_{1}}^{(1)}-1}, q_{2}^{-k_{h_{2}}^{(1)}-1}\right) e^{-(\log x)^{(k+1) \delta}}\right) \\
\gg & \max \left(q_{1}^{-k_{h_{1}}^{(1)}-1}, q_{2}^{-k_{h_{2}}^{(1)}-1}\right) e^{-C(\log \log x)^{2}} .
\end{aligned}
$$


Thus, we again have

$$
\left|\frac{1}{x} \sum_{n<x} e\left(\left(A_{1} \mathbf{m}_{1} \cdot \mathbf{v}_{1}+A_{2} \mathbf{m}_{2} \cdot \mathbf{v}_{2}\right) n\right)\right| \ll(\log x)^{-\lambda}
$$

for any given $\lambda>0$.

In general, we assume that for some $s_{l}(l=1,2)$,

$$
k_{j+1}^{(l)}-k_{j}^{(l)}<(\log x)^{k \delta} \quad\left(j<s_{l}\right)
$$

and

$$
k_{s_{l}+1}^{(l)}-k_{s_{l}}^{(l)} \geq(\log x)^{(k+1) \delta} .
$$

Here we set

$$
\bar{m}_{l}=A_{l} \sum_{j=1}^{s_{l}} m_{j}^{(l)} q_{l}^{k_{s_{l}}^{(l)}-k_{j}^{(l)}} \quad(l=1,2) .
$$

Then we have (as in the first case) $\bar{m}_{l} \not \equiv 0 \bmod q_{l}$ and $\log \left|\bar{m}_{l}\right| \ll(\log x)^{k \delta}$. Furthermore, we can estimate the sums

$$
\sum_{j=s_{l}+1}^{h_{l}} \frac{m_{j}^{(l)}}{q_{l}^{k_{j}^{(l)}+1}}=O\left((\log x)^{2 \lambda_{0}} q_{l}^{-(\log x)^{(k+1) \delta}}\right) .
$$

Thus we get

$$
\begin{aligned}
\mid A_{1} \mathbf{m}_{1} \cdot & \mathbf{v}_{1}+A_{2} \mathbf{m}_{2} \cdot \mathbf{v}_{2} \mid \\
\geq & \left|\frac{\bar{m}_{1}}{q_{1}^{k_{s_{1}}^{(1)}+1}}+\frac{\bar{m}_{2}}{q_{2}^{k_{s_{2}}^{(2)}}+1}\right|-\left|\sum_{j_{1}=s_{1}+1}^{h_{1}} \frac{m_{j_{1}}^{(1)}}{q_{1}^{(1)}+1}\right|-\left|\sum_{j_{2}=s_{2}+1}^{h_{2}} \frac{m_{j_{2}}^{(2)}}{k_{j_{2}}^{(2)}+1}\right| \\
\geq & \max \left(q_{1}^{-k_{s_{1}}^{(1)}-1}, q_{2}^{-k_{s_{2}}^{(1)}-1}\right) e^{-C \log \log x(\log x)^{k \delta}} \\
& -O\left((\log x)^{2 \lambda_{0}} \max \left(q_{1}^{-k_{s_{1}}^{(1)}-1}, q_{2}^{-k_{s_{2}}^{(1)}-1}\right) e^{-(\log x)^{(k+1) \delta}}\right) \\
\gg & \max \left(q_{1}^{-k_{s_{1}}^{(1)}-1}, q_{2}^{-k_{s_{2}}^{(1)}-1}\right) e^{-C \log \log x(\log x)^{k \delta}},
\end{aligned}
$$

which again implies (4.3).

Hence, we finally get

$$
\begin{array}{r}
\sum_{\left(\mathbf{m}_{1}, \mathbf{m}_{2}\right) \neq \mathbf{0}}\left|T_{\mathbf{m}_{1}, \mathbf{m}_{2}}\right| \cdot\left|\frac{1}{x} \sum_{n<x} e\left(\left(A_{1} \mathbf{m}_{1} \cdot \mathbf{v}_{1}+A_{2} \mathbf{m}_{2} \cdot \mathbf{v}_{2}\right) n\right)\right| \\
=O\left((\log x)^{-\lambda_{0}}\right)+O\left((\log x)^{4 \lambda_{0}-\lambda}\right),
\end{array}
$$

which completes the proof of Proposition 2.

5. Proof of Theorem 4. The proof of Theorem 4 relies on a direct application of proper saddle point approximations. 
Set

$$
a_{k_{1} k_{2}}=\#\left\{n<x \mid s_{q_{1}}(n)=k_{1}, s_{q_{2}}(n)=k_{2}\right\} .
$$

Then the empirical characteristic function is given by

$$
\varphi_{x}\left(t_{1}, t_{2}\right)=\frac{1}{x} \sum_{n<x} e^{i t_{1} s_{q_{1}}(n)+i t_{2} s_{q_{2}}(n)}=\frac{1}{x} \sum_{k_{1}, k_{2} \geq 0} a_{k_{1} k_{2}} e^{i t_{1} k_{1}+i t_{2} k_{2}},
$$

which implies that the numbers $a_{k_{1} k_{2}}$ can be determined by

$$
a_{k_{1} k_{2}}=\frac{1}{(2 \pi)^{2}} \int_{-\pi}^{\pi} \int_{-\pi}^{\pi} \varphi_{x}\left(t_{1}, t_{2}\right) e^{-i t_{1} k_{1}-i t_{2} k_{2}} d t_{1} d t_{2} .
$$

We first use Theorem 2 to extract the asymptotic leading term of $a_{k_{1} k_{2}}$. In fact, we need a little bit more general property.

Lemma 7 . Set

$$
M_{l}(x):=\frac{q_{l}-1}{2} \log _{q_{l}} x \quad \text { and } \quad D_{l}(x):=\frac{q_{l}^{2}-1}{12} \log _{q_{l}} x
$$

and let $P(x)$ denote the linear polynomial $P(x)=\operatorname{lcm}\left(q_{1}-1, q_{2}-1\right) x+B$ for some integer $B$ with $0 \leq B<\operatorname{lcm}\left(q_{1}-1, q_{2}-1\right)$. Then, for every $\varepsilon>0$ there exists $x_{0}=x_{0}(\varepsilon)$ such that

$$
\begin{aligned}
& \mid \frac{1}{x} \sum_{n<x} e^{i t_{1} s_{q_{1}}(P(n))+i t_{2} s_{q_{2}}(P(n))} \\
& \quad-e^{i\left(t_{1} M_{q_{1}}(x)+t_{2} M_{q_{2}}(x)\right)-\frac{1}{2}\left(t_{1}^{2} D_{q_{1}}^{2}(x)+t_{2}^{2} D_{q_{2}}^{2}(x)\right)} \mid<\varepsilon
\end{aligned}
$$

for all $x \geq x_{0}$ and for all $t_{1}, t_{2}$ real.

Proof. First we notice that Theorem 2 cannot be directly applied. It may occur that the leading term $A=\operatorname{lcm}\left(q_{1}-1, q_{2}-1\right)$ of $P(x)$ is not coprime to $q_{1}$ resp. to $q_{2}$. However, if $A=q_{l}^{K_{l}} \bar{A}_{l}$ (for some $K_{l}>0$ and $\bar{A}_{l}$ coprime to $q_{l}$ ) and if $B_{l}$ has $q_{l}$-ary expansion $B_{l}=B_{0}+B_{1} q_{l}+\ldots+B_{L_{l}} q_{l}^{L_{l}}$ then

$$
\begin{aligned}
s_{q_{l}}(A n+B) & =s_{q_{l}}\left(q_{l}^{K_{l}} \bar{A}_{l} n+B_{0}+B_{1} q_{l}+\ldots+B_{L_{l}} q_{l}^{L_{l}}\right) \\
& =s_{q_{l}}\left(q_{l}^{K_{l}-1} \bar{A}_{l} n+B_{1}+B_{2} q_{l}+\ldots+B_{L_{l}} q_{l}^{L_{l}-1}\right)+B_{0} \\
& =s_{q_{l}}\left(q_{l}^{K_{l}-2} \bar{A}_{l} n+B_{2}+B_{3} q_{l}+\ldots+B_{L_{l}} q_{l}^{L_{l}-2}\right)+B_{0}+B_{1} \\
& \vdots \\
& =s_{q_{l}}\left(\bar{A}_{l} n+\bar{B}_{l}\right)+\bar{C}_{l}
\end{aligned}
$$

for some integers $\bar{B}_{l}, \bar{C}_{l}$. Therefore, the joint (normalized) limiting distribution of $\left(s_{q_{1}}(A n+B), s_{q_{2}}(A n+B)\right)$ is the same as that of $\left(s_{q_{1}}\left(\bar{A}_{1} n+\bar{B}_{1}\right)\right.$, $\left.s_{q_{2}}\left(\bar{A}_{2} n+\bar{B}_{2}\right)\right)$, and $\bar{A}_{l}$ is coprime to $q_{l}, l=1,2$. Hence, we can always apply Theorem 2 for properly chosen linear polynomials $P_{l}(x), l=1,2$. 
By Levi's theorem it now follows from Theorem 2 (and the above remark) that for every fixed $t_{1}, t_{2}$ we have, as $x \rightarrow \infty$,

$$
\begin{aligned}
& \frac{1}{x} \sum_{n<x} e^{i\left(t_{1} s_{q_{1}}(P(n))+t_{2} s_{q_{2}}(P(n))\right) / \sqrt{\log x}} \\
& -e^{i\left(t_{1} M_{1}(x)+t_{2} M_{q_{2}}(x)\right) / \sqrt{\log x}-\frac{1}{2}\left(t_{1}^{2} D_{1}^{2}(x)+t_{2}^{2} D_{2}^{2}(x)\right) /(\log x)} \rightarrow 0 .
\end{aligned}
$$

Moreover, we can show that this convergence is uniform for all $t_{1}, t_{2}$. Since $\Phi\left(y_{1}\right) \Phi\left(y_{2}\right)$ is continuous we know that the normalized empirical distribution function

$$
\widetilde{F}_{x}\left(y_{1}, y_{2}\right):=\frac{1}{x} \#\left\{n<x \mid s_{q_{l}}(n) \leq M_{l}(n)+y_{l} D_{l}(x), l=1,2\right\}
$$

converges uniformly to $\Phi\left(y_{1}\right) \Phi\left(y_{2}\right)$. Furthermore, the variances

$$
\frac{1}{x} \sum_{n<x} \frac{\left(s_{q_{l}}(n)-M_{l}(n)\right)^{2}}{D_{l}^{2}(x)}
$$

are bounded (compare with (1.1)). Hence we get

$$
\int_{\max \left\{\left|y_{1}\right|,\left|y_{2}\right|\right\} \geq A} d \widetilde{F}_{x}\left(y_{1}, y_{2}\right) \ll \frac{1}{A} .
$$

Thus it follows by elementary means (and by using the definition of the characteristic function) that the convergence in (5.1) is uniform.

The proof of Theorem 2 will also make use of the following estimate on exponential sums.

Proposition 3. Let $q_{1}, \ldots, q_{d}>1$ be pairwise coprime integers. Then there exists a constant $c>0$ such that for all real numbers $t_{1}, \ldots, t_{d}$,

$$
\left|\frac{1}{x} \sum_{n<x} e\left(t_{1} s_{q_{1}}(n)+t_{2} s_{q_{2}}(n)+\ldots+t_{d} s_{q_{d}}(n)\right)\right| \ll e^{-c \log x \sum_{l=1}^{d}\left\|\left(q_{l}-1\right) t_{l}\right\|^{2}},
$$

where $\|t\|=\min _{k \in \mathbb{Z}}|t-k|$ denotes the distance to the integers.

A proof of Proposition 3 can be found in [7]. It is, more or less, a slight generalization of a corresponding estimate of exponential sums presented by Kim [18].

Now we can start with the proof of Theorem 4 .

Proof. For any $K>0$ and integers $s_{1}, s_{2}$ set

$C_{K}\left(s_{1}, s_{2}\right):=\left\{\left(t_{1}, t_{2}\right) \in[-\pi, \pi]^{2}:\left|t_{l}-\frac{2 \pi s_{l}}{q_{l}-1} \bmod 2 \pi\right| \leq \frac{K}{\sqrt{\log x}}, l=1,2\right\}$. 
Furthermore set

$$
A_{K}:=[-\pi, \pi]^{2} \backslash \bigcup_{s_{1}=0}^{q_{1}-2} \bigcup_{s_{2}=0}^{q_{2}-2} C_{K}\left(s_{1}, s_{2}\right) .
$$

By Proposition 3 for every $\varepsilon>0$ there exists $K=K(\varepsilon)$ such that

$$
\frac{1}{(2 \pi)^{2}} \int_{A_{K}}\left|\varphi_{x}\left(t_{1}, t_{2}\right)\right| d t_{1} d t_{2} \leq \frac{\varepsilon}{\log x} .
$$

Furthermore, we can choose $K \leq c^{\prime}(-\log \varepsilon)^{1 / 2}$ (for some constant $c^{\prime}>0$ ). So it remains to consider the integrals

$$
\begin{aligned}
& I_{K}\left(s_{1}, s_{2}\right):=\frac{1}{(2 \pi)^{2}} \int_{C_{K}\left(s_{1}, s_{2}\right)}\left(\frac{1}{x} \sum_{n<x} e^{i t_{1}\left(s_{q_{1}}(n)-k_{1}\right)+i t_{2}\left(s_{q_{2}}(n)-k_{2}\right)}\right) d t_{1} d t_{2} \\
& =e^{-2 \pi i\left(k_{1} \frac{s_{1}}{q_{1}-1}+k_{2} \frac{s_{2}}{q_{2}-1}\right)} \frac{1}{(2 \pi)^{2}} \\
& \quad \times \int_{C_{K}(0,0)}\left(\frac{1}{x} \sum_{n<x} e^{i t_{1}^{\prime}\left(s_{q_{1}}(n)-k_{1}\right)+i t_{2}^{\prime}\left(s_{q_{2}}(n)-k_{2}\right)}\right) e^{2 \pi i\left(\frac{s_{1}}{q_{1}-1}+\frac{s_{2}}{q_{2}-1}\right) n} d t_{1}^{\prime} d t_{2}^{\prime} .
\end{aligned}
$$

By Lemma 7 it is easy to evaluate $I_{K}(0,0)$ asymptotically. For sufficiently large $x \geq x_{0}(\varepsilon)$ we have

$$
\left|\varphi_{x}\left(t_{1}, t_{2}\right)-e^{i\left(t_{1} M_{1}(x)+t_{2} M_{2}(x)\right)-\frac{1}{2}\left(t_{1}^{2} D_{1}^{2}(x)+t_{2}^{2} D_{2}^{2}(x)\right)}\right|<\varepsilon
$$

for all real $t_{1}, t_{2}$, and consequently

$$
\begin{aligned}
I_{K}(0,0) & \frac{1}{(2 \pi)^{2}} \int_{C_{K}(0,0)} e^{i t_{1}\left(M_{1}(x)-k_{1}\right)+i t_{2}\left(M_{2}(x)-k_{2}\right)-\frac{1}{2}\left(t_{1}^{2} D_{1}^{2}(x)+t_{2}^{2} D_{2}^{2}(x)\right)} d t_{1} d t_{2} \\
& +O\left(\frac{\varepsilon K^{2}}{\log x}\right) \\
= & \frac{1}{(2 \pi)^{2}} \int_{-\infty}^{\infty} \int_{-\infty}^{\infty} e^{i t_{1}\left(M_{1}(x)-k_{1}\right)+i t_{2}\left(M_{2}(x)-k_{2}\right)-\frac{1}{2}\left(t_{1}^{2} D_{1}^{2}(x)+t_{2}^{2} D_{2}^{2}(x)\right)} d t_{1} d t_{2} \\
& +O\left(\frac{\varepsilon(-\log \varepsilon)}{\log x}\right) \\
= & \prod_{l=1}^{2}\left(\frac{1}{\sqrt{2 \pi} D_{q_{l}}(x)} \exp \left(-\frac{\left(k_{l}-M_{q_{l}}(x)\right)^{2}}{2 D_{q_{l}}^{2}(x)}\right)\right)+O\left(\frac{\varepsilon(-\log \varepsilon)}{\log x}\right) .
\end{aligned}
$$

In order to treat the remaining integrals $I_{K}\left(s_{1}, s_{2}\right)$ we recall that $d$ and $A$ denote $d=\operatorname{gcd}\left(q_{1}-1, q_{2}-1\right)$ and $A=\operatorname{lcm}\left(q_{1}-1, q_{2}-1\right)$. We represent 
$s_{1}, s_{2}$ by

$$
s_{l}=m_{l} \frac{q_{l}-1}{d}+r_{l} \quad\left(0 \leq m_{l}<d, 0 \leq r_{l}<\left(q_{l}-1\right) / d, l=1,2\right)
$$

and observe that

$$
\begin{aligned}
\frac{s_{1}}{q_{1}-1}+\frac{s_{2}}{q_{2}-1} & =\frac{m_{1}+m_{2}}{d}+\frac{r_{1}}{q_{1}-1}+\frac{r_{2}}{q_{2}-1} \\
& =\frac{m_{1}+m_{2}}{d}+\frac{r_{1} \frac{q_{2}-1}{d}+r_{2} \frac{q_{1}-1}{d}}{A} .
\end{aligned}
$$

Thus, $\zeta:=e^{2 \pi i\left(\frac{s_{1}}{q_{1}-1}+\frac{s_{2}}{q_{2}-1}\right)}$ is always an $A$ th root of unity and $\zeta=1$ if and only if

$$
m_{1}+m_{2}=d, \quad r_{1}=0 \text { and } r_{2}=0 .
$$

Thus, if (5.3) is satisfied, i.e., $s_{1}=m_{1} \frac{q_{1}-1}{d}$ and $s_{2}=\left(d-m_{1}\right) \frac{q_{2}-1}{d}$, we have (recall that $k_{1} \equiv k_{2} \bmod d$ )

$$
I_{K}\left(s_{1}, s_{2}\right)=e^{-2 \pi i \frac{m_{1}}{d}\left(k_{1}-k_{2}\right)} I_{K}(0,0)=I_{K}(0,0) .
$$

Hence

$$
\sum_{m_{1}=0}^{d-1} I_{K}\left(m_{1} \frac{q_{1}-1}{d},\left(d-m_{1}\right) \frac{q_{2}-1}{d}\right)=d I_{K}(0,0)
$$

which fits (by (5.2)) the asymptotic leading term of $a_{k_{1} k_{2}}$.

Finally we have to consider the case where

$$
\zeta=e^{2 \pi i\left(\frac{s_{1}}{q_{1}-1}+\frac{s_{2}}{q_{2}-1}\right)} \neq 1 .
$$

Here we have

$$
\begin{aligned}
& I_{K}\left(s_{1}, s_{2}\right)=e^{-2 \pi i\left(k_{1} \frac{s_{1}}{q_{1}-1}+k_{2} \frac{s_{2}}{q_{2}-1}\right)} \\
& \times \sum_{B=0}^{A-1} \zeta^{B} \int_{C_{K}(0,0)}\left(\frac{1}{x} \sum_{n^{\prime}<(x-B) / A} e^{i t_{1}^{\prime}\left(s_{q_{1}}\left(A n^{\prime}+B\right)-k_{1}\right)+i t_{2}^{\prime}\left(s_{q_{2}}\left(A n^{\prime}+B\right)-k_{2}\right)}\right) d t_{1}^{\prime} d t_{2}^{\prime} .
\end{aligned}
$$

As above, it follows by Lemma 7 that for sufficiently large $x \geq x_{1}(\varepsilon)$ (and of course uniformly for all $B=0,1, \ldots, A-1$ )

$$
\begin{aligned}
\int_{C_{K}(0,0)}\left(\frac{1}{x} \sum_{n^{\prime}<(x-B) / A} e^{i t_{1}^{\prime}\left(s_{q_{1}}\left(A n^{\prime}+B\right)-k_{1}\right)+i t_{2}^{\prime}\left(s_{q_{2}}\left(A n^{\prime}+B\right)-k_{2}\right)}\right) d t_{1}^{\prime} d t_{2}^{\prime} \\
\quad=\frac{1}{A} \prod_{l=1}^{2}\left(\frac{1}{\sqrt{2 \pi} D_{q_{l}}(x)} \exp \left(-\frac{\left(k_{l}-M_{q_{l}}(x)\right)^{2}}{2 D_{q_{l}}^{2}(x)}\right)\right)+O\left(\frac{\varepsilon \log (-\varepsilon)}{\log x}\right) .
\end{aligned}
$$

Thus

$$
I_{K}\left(s_{1}, s_{2}\right)=O\left(\frac{\varepsilon(-\log \varepsilon)}{\log x}\right) .
$$

This completes the proof of Theorem 4. 
Acknowledgements. The author is indebted to Cecile Dartyge for pointing out the possible use of [1] to describe the joint distribution of $q$ additive functions. This hint was the key to all major results of this paper. The author also wants to thank Adolf J. Hildebrand for several discussions on this topic.

\section{References}

[1] N. L. Bassily and I. Kátai, Distribution of the values of q-additive functions on polynomial sequences, Acta Math. Hungar. 68 (1995), 353-361.

[2] J. Bésineau, Indépendence statistique d'ensembles liés à la fonction "sommes des chiffres", Acta Arith. 20 (1972), 401-416.

[3] J. Coquet, Corrélation de suites arithmétiques, Sém. Delange-Pisot-Poitou, 20e Année 1978/79, Exp. 15, 12 p. (1980).

[4] H. Delange, Sur les fonctions q-additives ou q-multiplicatives, Acta Arith. 21 (1972), 285-298.

[5] - Sur la fonction sommatoire de la fonction "somme des chiffres", Enseign. Math. 21 (1975), 31-47.

[6] M. Drmota and J. Gajdosik, The distribution of the sum-of-digits function, J. Théor. Nombres Bordeaux 10 (1998), 17-32.

[7] M. Drmota and G. Larcher, The sum-of-digits function and uniform distribution modulo 1, J. Number Theory, to appear.

[8] J. M. Dumont and A. Thomas, Gaussian asymptotic properties of the sum-of-digits functions, ibid. 62 (1997), 19-38.

[9] E. Fouvry et C. Mauduit, Sommes des chiffres et nombres presque premiers, Math. Ann. 305 (1996), 571-599.

[10] —, - Méthodes de crible et fonctions sommes des chiffres, Acta Arith. 77 (1996), $339-351$.

[11] A. O. Gelfond, Sur les nombres qui ont des propriétés additives et multiplicatives données, ibid. 13 (1968), 259-265.

[12] P. J. Grabner, P. Kirschenhofer, H. Prodinger, and R. F. Tichy, On the moments of the sum-of-digits function, in: Applications of Fibonacci Numbers 5, Kluwer, 1993, 263-271.

[13] P. J. Grabner, P. Liardet and R. F. Tichy, work in preparation.

[14] A. J. Hildebrand, personal communication.

[15] L. K. Hua, Additive Theory of Prime Numbers, Transl. Math. Monographs 13, Amer. Math. Soc., Providence, 1965.

[16] I. Kátai, Distribution of q-additive function, in: Probability Theory and Applications, Essays to the Memory of J. Mogyorodi, Math. Appl. 80, Kluwer, Dordrecht, 1992, 309-318.

[17] R. E. Kennedy and C. N. Cooper, An extension of a theorem by Cheo and Yien concerning digital sums, Fibonacci Quart. 29 (1991), 145-149.

[18] D.-H. Kim, On the joint distribution of q-additive functions in residue classes, J. Number Theory 74 (1999), 307-336.

[19] P. Kirschenhofer, On the variance of the sum of digits function, in: Lecture Notes in Math. 1452, Springer, 1990, 112-116. 
[20] E. Manstavičius, Probabilistic theory of additive functions related to systems of numerations, in: Analytic and Probabilistic Methods in Number Theory, VSP, Utrecht, 1997, 413-430.

[21] A. Pethő and R. F. Tichy, S-unit equations, linear recurrences and digit expansions, Publ. Math. Debrecen 42 (1993), 145-154.

[22] H. P. Schlickewei, S-unit equations over number fields, Invent. Math. 102 (1990), 95-107.

[23] —, Linear equations in integers with bounded sum of digits, J. Number Theory 35 (1990), 335-344.

[24] J. Schmid, The joint distribution of the binary digits of integer multiples, Acta Arith. 43 (1984), 391-415.

[25] W. M. Schmidt, The joint distribution of the digits of certain integer s-tuples, in: Studies in Pure Mathematics in Memory of P. Turán, Birkhäuser, 1983, 605-622.

[26] H. G. Senge and E. G. Straus, PV-numbers and sets of multiplicity, Period. Math. Hungar. 3 (1973), 93-100.

[27] J. A. Solinas, On the joint distribution of digital sums, J. Number Theory 33 (1989), $132-151$.

[28] C. L. Stewart, On the representation of an integer in two different bases, J. Reine Angew. Math. 319 (1980), 63-72.

[29] M. Waldschmidt, Minorations de combinaisons linéaires de logarithmes de nombres algébriques, Canad. J. Math. 45 (1993), 176-224.

Department of Geometry

Technische Universität Wien

Wiedner Hauptstraße 8-10/113

A-1040 Wien, Austria

E-mail: michael.drmota@tuwien.ac.at

Received on 18.2.2000

and in revised form on 1.2.2001 